\title{
Arbitrariness of Jordan Structure in Factorization: the Geometric Multiplicity Restriction and the 3-by-3 Case *
}

\author{
Charles R. Johnson ${ }^{1}$, Drew Lewis ${ }^{2}$, Yulin Zhang $^{3 \dagger}$ \\ ${ }^{1}$ Department of Mathematics, College of William and Mary, Williamsburg, VA, 23187 \\ crjohnso@math.um.edu \\ 2 Department of Mathematics, University of Alabama \\ andrew.m.lewis@ua.edu \\ ${ }^{3}$ Centro de Matemática, Universidade do Minho, 4710 Braga, Portugal \\ zhang@math.uminho.pt
}

9th November 2012

\begin{abstract}
For the problem of which Jordan forms are possible for $n$-by- $n$ complex matrices $A, B$ and $C$, when $A=B C$, geometric multiplicity restrictions are given for the eigenvalues of the three matrices. Together with the obvious determinantal condition on the eigenvalues, these necessary conditions are shown to be sufficient for the problem when $n<4$, but not for $n \geq 4$. Some basic observations about the problem are given in the process.
\end{abstract}

Keywords: Jordan form; geometric multiplicity; matrix product.

AMS Classifications: 15A23

\section{Introduction}

Suppose that an invertible matrix $A \in M_{n}(\mathbb{C})$ is given, so that we may regard its Jordan canonical form (JCF) as known. Then, among all the

${ }^{*}$ This study was supported in part by National Science Foundation Grant DMS-03-53510,USA, CMAT through the FCT Pluriannual Funding Program and PTDC/MAT/112273/2009, Portugal.

${ }^{\dagger}$ Corresponding author 
ways of factoring $A$ as $A=B C$, with $B, C \in M_{n}(\mathbb{C})$, how arbitrary may the JCF's of $A, B$ and $C$ be taken to be? Equivalently, when $A=B C$, what are all the possibilities for the triple of JCF's of $A, B, C$ ? This is known to be a very difficult problem, in general, but a number of prior works discuss portions of it, some explicitly and some very theoretically [2], 6], [7, [8], [9], [10]. Perhaps the first of these [1, shows that if the JCF aspect of the problem is weakened to asking only what the 3 spectra can be, there is no restriction, save the obvious condition that $\operatorname{det} A=\operatorname{det} B \operatorname{det} C$; in fact $A$ maybe taken arbitrarily (as long as it is not scalar) and the spectra of $B$ and $C$ chosen freely, so long as the determinant condition is met. This has been specialized by showing that, in addition, $B$ and $C$ may be chosen to be nonderogatory [4, [6], so that a particular JCF is attainable in all cases. At the other extreme, choosing with diagonalizability can be more difficult. The 4-by-4 case has been sorted out in [5] and a theoretical approach has been given in [5]. A result to be given here explains this somewhat.

The 1-by-1 and 2-by-2 cases of our problem are relatively straightforward. Here, we identify a more subtle necessary condition based upon geometric multiplicity. This allows complete solution of the problem in the 3-by-3 case, but unfortunately, not in general. First, we make a few observations.

\section{Observations}

Because the determinant is the product of the eigenvalues, the most obvious restriction on our problem, that

$$
\operatorname{det} A=\operatorname{det} B \operatorname{det} C,
$$

may be viewed as a numerical restriction on the eigenvalues in the JCF's of $A, B$ and $C$. If those eigenvalues, including multiplicities are $A: \alpha_{1}, \alpha_{2}, \ldots, \alpha_{n}$; $B: \beta_{1}, \beta_{2}, \ldots, \beta_{n}$; and $C: \gamma_{1}, \gamma_{2}, \ldots, \gamma_{n}$, we have the determinant condition

Lemma 1 If $A=B C$, then $\prod_{i=1}^{n} \alpha_{i}=\prod_{j=1}^{n} \beta_{j} \gamma_{j}$.

In case $n=1$, the determinant condition is necessary and sufficient. If $n=2$, it is also necessary and sufficient, unless at least one of the three matrices has two equal eigenvalues and is diagonalizable (and thus is a scalar matrix). This situation is covered by our necessary condition of the next section. 
Another useful observation is the fact that our problem is symmetric in the three matrices $A, B$ and $C$. Since all three matrices must be invertible, we may also write, for example,

$$
B=A C^{-1} \text {. }
$$

Since the JCF of $C^{-1}$ is determined by that of $C$, and since $A C^{-1}$ is similar to $C^{-1} A$, it is equivalent to take any of the three matrices as the left hand side and the other two matrices in either order.

Finally, as the JCF is similarity invariant, and similarity may be passed through the equation (e.g. $S^{-1} A S=S^{-1} B S S^{-1} C S$ ), we may take, without loss of generality, any one of the three matrices to actually be in JCF.

\section{The Geometric Multiplicity Restriction}

For $A \in M_{n}(\mathbb{C})$, denote the geometric multiplicity of an eigenvalue $\lambda$ of $A$ by $g_{A}(\lambda)$, i.e., $g_{A}(\lambda)=n-\operatorname{rank}(A-\lambda I)$. Of course $g_{A}(\lambda)=0$ means that $\lambda$ is not an eigenvalue of $A$. We then have the following general constraint on our 3 - matrix JCF problem.

Theorem 1 If $A, B, C \in M_{n}(\mathbb{C}), A$ is nonsingular and $A=B C$, then

(i) $g_{A}(\beta \gamma) \geq g_{B}(\beta)+g_{C}(\gamma)-n$ and

(ii) $g_{B}\left(\frac{\alpha}{\gamma}\right) \geq g_{A}(\alpha)+g_{C}(\gamma)-n$.

Proof. Of course, by symmetry, (ii) is the same statement as (i), so that it suffices to verify (i).

Let $R=B-\beta I$ and $S=C-\gamma I$, with $R, S \in M_{n}(\mathbb{C})$. We then obtain that $B=R+\beta I$ and $C=S+\gamma I$, and, thus,

$$
B C=(R+\beta I)(S+\gamma I)=R S+\gamma R+\beta S+\beta \gamma I
$$

which gives

$$
B C-\beta \gamma I=R(S+\gamma I)+\beta S .
$$

Taking the rank of both sides, we get

$$
\operatorname{rank}(B C-\beta \gamma I) \leq \operatorname{rank}(R)+\operatorname{rank}(S) .
$$

Replace $R$ and $S$ by $B-\beta I$ and $C-\gamma I$, and we have

$$
\operatorname{rank}(B C-\beta \gamma I) \leq \operatorname{rank}(B-\beta I)+\operatorname{rank}(C-\gamma I) .
$$


Since $g_{T}(\lambda)=n-\operatorname{rank}(T-\lambda I)$, we have

$$
g_{B C}(\beta \gamma) \geq g_{B}(\beta)+g_{C}(\gamma)-n,
$$

which is (i). This completes the proof.

We refer to the constraint placed upon our problem by Theorem 1 as the geometric multiplicity restriction (GMR). It explains, in part, why the nonderogatory refinement of Sourour's result is natural and why the diagonalizable case of our problem can be more difficult. If two of the matrices have sufficiently highly geometrically multiple eigenvalues, then the third has to have a certain eigenvalue, i.e., a local version of the constraint given by Lemma 1.

Now, the solution to our problem may be completed when $n=2$. If none of the matrices has a double eigenvalue and is diagonalizable, then the determinant condition is necessary and sufficient. If at least one does, then the GMR applies and at least one eigenvalue from each JCF must satisfy a product equation. It is then easy to show that the determinant condition and GMR are sufficient. This may also be verified by calculation without explicitly observing the GMR.

\section{The 3-by-3 Case}

We may now go further by giving a complete solution to our problem in the 3-by-3 case. We do this by considering all possible triples of JCF's that satisfy the determinant condition. The list that explicitly need be considered is limited by the symmetry of the problem. Some are ruled out by the GMR, and we show that the remaining ones may be constructed, some by known results and others by construction here. This means that the determinant condition and GMR are necessary and sufficient in the 3by-3 case. Unfortunately, this does not remain valid when $n=4$, as shown

by examples resulting from the analysis of [5]. Most cases not ruled out by [5] and the determinant condition and GMR are constructable for $n=4$.

Now, let us look at the JCF of a 3-by-3 matrix. When one of the three matrices is scalar, then the other two matrices must have the same Jordan structure. Indeed, if $A$ is scalar, then, $C$ must be multiple of $B^{-1}$, and if $B$ is scalar, then, $A$ must be multiple of $C$. So, the nonscalar condition is necessary. There are only 5 JCF's a matrix can take such that are nonscalar and nonsingular. Three of them are nonderogatory (labeled by $N_{1}, N_{2}, N_{3}$ ) and two are not (labeled by $T_{1}, T_{2}$ ). We assume that $\alpha, \beta$ and $\gamma$ are all 
distinct and nonzero, and $\lambda, \mu$ are also distinct and nonzero. Then the labels are as follows:

$$
\begin{gathered}
N_{1}=\left[\begin{array}{ccc}
\alpha & 0 & 0 \\
0 & \beta & 0 \\
0 & 0 & \gamma
\end{array}\right], N_{2}=\left[\begin{array}{ccc}
\alpha & 1 & 0 \\
0 & \alpha & 0 \\
0 & 0 & \beta
\end{array}\right], N_{3}=\left[\begin{array}{ccc}
\alpha & 1 & 0 \\
0 & \alpha & 1 \\
0 & 0 & \alpha
\end{array}\right], \\
T_{1}=\left[\begin{array}{ccc}
\lambda & 0 & 0 \\
0 & \lambda & 0 \\
0 & 0 & \mu
\end{array}\right], T_{2}=\left[\begin{array}{ccc}
\lambda & 1 & 0 \\
0 & \lambda & 0 \\
0 & 0 & \lambda
\end{array}\right] .
\end{gathered}
$$

When two of the three matrices are nonderogatory, then the sufficiency is implied by [4. The cases $N_{3} T_{1} T_{2}$ and $T_{2} T_{2} T_{1}$ are excluded by the GMR in combination with the determinant restriction. So, there are only eleven triples we need to consider. They are: (1) $N_{1} T_{1} T_{1}$, (2) $N_{1} T_{1} T_{2}$, (3) $N_{1} T_{2} T_{2}$, (4) $N_{2} T_{1} T_{1}$, (5) $N_{2} T_{1} T_{2}$, (6) $N_{2} T_{2} T_{2}$, (7) $N_{3} T_{1} T_{1}$, (8) $N_{3} T_{2} T_{2}$, (9) $T_{1} T_{1} T_{1}$, (10) $T_{2} T_{1} T_{1}$, and (11) $T_{2} T_{2} T_{2}$.

Theorem 2 Let $n=3$ and suppose that the 3 JCF's, with nonzero eigenvalues, $J_{1}, J_{2}, J_{3}$ are given. Then, there exist matrices $A, B, C \in M_{3}(\mathbb{C})$ such that $J C F(A)=J_{1}, J C F(B)=J_{2}$, and $J C F(C)=J_{3}$, and $A=B C$, if and only if the determinant condition and GMR are satisfied.

Proof. Because of the symmetry of the problem, we assume, without loss of generality, that $A$ is the only nonderogatory matrix, if any. The other two matrices in all cases have an eigenvalue with geometric multiplicity two, so an eigenvalue is forced by the GMR. Thus, we can assume without loss of generality that an eigenvalue of $C$ must be $\frac{\alpha}{\lambda}$.

We first deal with the cases (7) and (8), i.e., the $\operatorname{JCF}(A)$ is $N_{3}$. In fact, a simple calculation may give us the desired Jordan form. For $(7)$,

$$
A \cong\left[\begin{array}{ccc}
\alpha & \lambda & 0 \\
0 & \alpha & \frac{\alpha}{\lambda} \\
0 & 0 & \alpha
\end{array}\right]=\left[\begin{array}{ccc}
\lambda & 0 & 0 \\
0 & \mu & 1 \\
0 & 0 & \lambda
\end{array}\right]\left[\begin{array}{ccc}
\frac{\alpha}{\lambda} & 1 & 0 \\
0 & \frac{\alpha}{\mu} & 0 \\
0 & 0 & \frac{\alpha}{\lambda}
\end{array}\right]=B C
$$

and for case (8),

$$
A \cong\left[\begin{array}{ccc}
\alpha & \frac{\alpha}{\lambda} & 1 \\
0 & \alpha & \lambda \\
0 & 0 & \alpha
\end{array}\right]=\left[\begin{array}{ccc}
\lambda & 1 & 0 \\
0 & \lambda & 0 \\
0 & 0 & \lambda
\end{array}\right]\left[\begin{array}{ccc}
\frac{\alpha}{\lambda} & 0 & 0 \\
0 & \frac{\alpha}{\lambda} & 1 \\
0 & 0 & \frac{\alpha}{\lambda}
\end{array}\right]=B C
$$


For the other cases, we first show that we can get matrices with required Jordan structure by restricting to the following form:

$$
A=\left[\begin{array}{lll}
\alpha & 0 & 0 \\
0 & a & b \\
0 & c & d
\end{array}\right]=\left[\begin{array}{ccc}
\lambda & 0 & 0 \\
0 & * & * \\
0 & * & *
\end{array}\right]\left[\begin{array}{ccc}
\frac{\alpha}{\lambda} & 0 & 0 \\
0 & * & * \\
0 & * & *
\end{array}\right]=B C
$$

When $\operatorname{JCF}(A)=N_{1}$, or $\operatorname{JCF}(A)=N_{2}$ but the eigenvalue with algebraic multiplicity 1 is the forced eigenvalue, this is easy to see. Suppose $J C F(A)=N_{2}$ and the eigenvalue with algebraic multiplicity 2 is the forced eigenvalue. Then, without loss of generality, we take $\sigma(A)=\{\alpha, \alpha, \beta\}$, $\sigma(B)=\{\lambda, \lambda, \mu\}$ and $\sigma(C)=\{\gamma, \gamma, \delta\}$, with $\alpha \neq \beta, \lambda$ and $\mu$ not necessarily distinct, and $\gamma$ and $\delta$ not necessarily distinct. We assume $\alpha=\lambda \gamma$, by the determinant restriction, we have that $(\lambda \gamma)^{2} \beta=\lambda^{2} \mu \gamma^{2} \delta$ and thus $\beta=\mu \delta$. So that, we can rewrite the required Jordan structure as

$$
A=\left[\begin{array}{lll}
\beta & 0 & 0 \\
0 & a & b \\
0 & c & d
\end{array}\right]=\left[\begin{array}{lll}
\mu & 0 & 0 \\
0 & * & * \\
0 & * & *
\end{array}\right]\left[\begin{array}{lll}
\delta & 0 & 0 \\
0 & * & * \\
0 & * & *
\end{array}\right]=B C .
$$

In any case, we can reduce the 3 -by- 3 case to the 2 -by- 2 case. We denote the lower-right 2-by-2 submatrices of $A, B, C$ by $A^{\prime}, B^{\prime}, C^{\prime}$, respectively. By having different values of $a, b, c$, and $d$, we can produce an $A$ with JCF $N_{1}, N_{2}, T_{1}$ or $T_{2}$. For a given $A$, we can choose the $B^{\prime}$ and $C^{\prime}$ (nonscalar) to get the desired eigenvalues to get the desired JCF of $B$ and $C$. When $B^{\prime}$ and $C^{\prime}$ cannot be chosen nonscalar, it happens only in case (4), and in $N_{2}$ the eigenvalue with algebraic multiplicity 2 is the forced eigenvalue, we rewrite the triples as

$$
A=\left[\begin{array}{ccc}
\alpha & 1 & 0 \\
0 & \alpha & 0 \\
0 & 0 & \beta
\end{array}\right] \cong\left[\begin{array}{ccc}
\alpha & \lambda & 0 \\
0 & \beta & \frac{\alpha}{\lambda} \\
0 & 0 & \alpha
\end{array}\right]=\left[\begin{array}{ccc}
\lambda & 0 & 0 \\
0 & \mu & 1 \\
0 & 0 & \lambda
\end{array}\right]\left[\begin{array}{ccc}
\frac{\alpha}{\lambda} & 1 & 0 \\
0 & \frac{\beta}{\mu} & 0 \\
0 & 0 & \frac{\alpha}{\lambda}
\end{array}\right]=B C
$$

This completes the proof.

Example Because of the results of [5], the following triple of JCF's is not realized by $A, B, C \in M_{4}(\mathbb{C})$, with $A=B C$, in spite of the fact that both the determinant condition and GMR are vacuously satisfied. For example

$$
A=\operatorname{diag}(2,2,3,3), \quad B=\operatorname{diag}(i, i,-2,-2) \text { and } C=\operatorname{diag}(-i,-i, 3,3) \text {. }
$$

(Direct verification would be quite complicated.) 


\section{References}

[1] A. Sourour, A Factorization Theorem for Matrices, Linear Multilinear 19 (1986), 141-147.

[2] R. A. Horn and C. R. Johnson, Topics in Matrix Analysis, Cambridge Univ.Press, 1991.

[3] C. R. Johnson and D. Lewis, Arbitrariness of Jordan Structure in Factorization, REU Report to the National Science Foundation, William and Mary, 2004. (preprint)

[4] C. R. Johnson and Yulin Zhang, Spectrally Arbitrary Factorization: The Nonderogatory Case. Textos de Matemática, Universidade de Coimbra, Série B, №39, 79-92, 2006.

[5] C. R. Johnson and Yulin Zhang, The Jordan Form Problem for $C=$ $A B$ : the Balanced, Diagonalizable Case, Asian-European Journal of Mathematics, vol.3, No.4 (2010) 607-621.

[6] A. Lev, Product of Cyclic Similarity Classes in the Group $G L_{n}(F)$, Lin. Alg. Appl. 202 (1994), 235-266.

[7] D. Carlson and E. Marques de Sá, Generalized minimax and interlacing theorem, Linear and Multilinear Algebra 15 (1984), 77-103.

[8] F. C. Silva, Sums and products of matrices with prescribed similarity classes, Linear and Multilinear Algebra 27 (1990), 317-323.

[9] F. C. Silva, The eigenvalues of the product of matrices with prescribed similarity classes, Linear and Multilinear Algebra 34 (1993), 269-277.

[10] YuLin Zhang, On the number of invariant polynomials of the product of matrices with prescribed similarity classes, Linear algebra and its Application 277 (1998), 253-269. 